\title{
THE ROLE OF DREB2 GENE IN DROUGHT TOLERANCE OF COMMON WHEAT (TRITICUM AESTIVUM L.) ASSOCIATED WITH AZOSPIRILLUM BRASILENSE
}

\author{
DeHghani, I. ${ }^{1,5}$ - MostaJerAn, A. ${ }^{2 *}-$ ESMAEILI, A. ${ }^{3}$ - GHANNADIAN, M. ${ }^{4,5}$ \\ ${ }^{I}$ University of Isfahan, Plant Science Division, Department of Biology, Faculty of Science \\ Isfahan, Iran \\ (phone: +98-91-39041386; fax: +98-31-37932456) \\ ${ }^{2}$ University of Isfahan, Plant Science Division, Department of Biology, Faculty of Science \\ Isfahan, Iran \\ (phone: +98-31-37932471; fax: +98-31-37932456) \\ ${ }^{3}$ University of Isfahan, Cell, Molecular Biology and Biochemistry Division \\ Department of Biology, Faculty of Sciences, Isfahan, Iran \\ (phone: +98-31-37932452; fax: +98-31-37932456) \\ ${ }^{4}$ Isfahan University of Medical Sciences, Isfahan Pharmaceutical Sciences Research Center \\ School of Pharmacy, Isfahan, Iran \\ (phone: +98-31-37921335; fax: +98-31-36680048) \\ ${ }^{5}$ Isfahan University of Medical Sciences, Department of Pharmacognosy, School of Pharmacy \\ Isfahan, Iran \\ (phone: +98-31-37921335; fax: +98-31-36680048) \\ *Corresponding author \\ e-mail: mostajerana@yahoo.com; phone: +98-31-37932471; fax: +98-31-37932456
}

(Received $31^{\text {st }}$ Jul 2018; accepted $27^{\text {th }}$ Feb 2019)

\begin{abstract}
Dehydration causes loss of wheat (Triticum aestivum L.) yield. Inoculation of wheat with Azospirillum brasilense improves its tolerance to drought. Although up-regulation of DREB2 gene has increased drought tolerance of wheat, less information exists about DREB2 expression under inoculation with $A$. brasilense. In this study, the physiological responses of different pairs of six wheat cultivars with A. brasilense $\mathrm{Sp} 7$ and $\mathrm{Sp} 245$ strains were evaluated to drought and the tolerant (Roshan-Sp245), sensitive (Shahpasand-Sp7), moderately tolerant (Roshan-Sp7) and moderately sensitive pairs (Shahpasand-Sp245) were selected. Afterward, in the second experiment, DREB2 expression of the selected pairs was evaluated under inoculation and/or dehydration $\left(\psi_{\mathrm{w}}=-90 \mathrm{MPa}\right)$ at 0,120 and 360 minutes. At $120 \mathrm{~min}$, $D R E B 2$ expression was more in the tolerant and moderately tolerant pairs than others and also higher in inoculated and/or dehydration conditions. Root's DREB2 was up-regulated almost 700-fold in the tolerant and moderately tolerant pairs under dehydration. In contrast, in the sensitive cv., DREB2 expression did not change due to dehydration or inoculation. This could be the effect of compatibility or homology of A. brasilense strains and wheat cultivars which affected DREB2 expression. Therefore, DREB2 expression had a significant effect on increased drought tolerance in tolerant and moderately tolerant pairs.
\end{abstract}

Keywords: wheat, Azospirillum brasilense, Sp7, Sp245, drought tolerance, DREB2 gene, dehydration

Abbreviations: DREB2 gene, DRE-binding factor gene; FC, Field capacity; PAL, Phenylalanine Ammonia lyase enzyme (EC 4.3.1.24); TAL, Tyrosine Ammonia lyase enzyme (EC 4.3.1.23); RDW, Root Dry Weight; SDW, Shoot Dry Weight; MSL, Maximum Shoot Length; ARL, Average Root Length; NMR, Number of Main Roots; NRB, Number of Root Branches; RTP, Root Total Protein; STP, Shoot Total Protein; RPAL, Root PAL activity; SPAL, Shoot PAL activity; RTAL, Root TAL activity; STAL, Shoot TAL activity 


\section{Introduction}

Drought is one of the major or more significant stresses which adversely affect the plant water status and crop yield (Kramer and Boyer, 1995; Bray, 2000). Dehydration or loss of water causes reduction of cell turgor pressure, an increase in negative water potential and consequently less plant production (Liu et al., 2005). Azospirillum brasilense is one of the PGPRs (Plant Growth Promoting Rhizobacteria) that can associate with wheat and increase crop quality and quantity through suitable physiological and biochemical modification in plants (Dobbelaere et al., 2003; ElSamad and Hetta, 2005; Pereyra et al., 2006; Hungria et al., 2010; Veresoglou and Menexes, 2010). The effect of PGPRs on dehydration responsive genes in plants has been evaluated but not well studied specifically about the DREB2 gene expression during inoculation of wheat with A. brasilense. Therefore, the objective of this study is to evaluate the effect of $A$. brasilense on DREB2 gene expression in wheat cultivars under dehydration condition.

\section{Review of Literature}

The ability of Azospirillum to establish an association system with plants such as wheat through which it promotes better growth and development (Dobereiner and Day, 1976; Bashan and Holguin, 1997) is due to its capacity for $\mathrm{N}_{2}$ fixation capacity (Bermner et al., 1995) and production of some bacterial compounds having plant phytohormone properties such as nitric oxide, IAA (indole-3-acetic acid) polyamines and amino acids (Hartmann et al., 1994; El-Khawas and Adachi, 1999; Pakdaman et al., 2014). These bacterial compounds produced in wheat rhizosphere caused structural changes in wheat roots, stimulated root growth and increased water and minerals absorption by the plant (Perrig et al., 2007; Amooaghaie et al., 2004; Casimiro et al., 2003; Correa-Aragunde et al., 2004). These advantages for plants are also reported at dehydration condition for different pairs of plant and bacteria especially at the early stage of growth (Bashan and Holguin, 1997; Hartmann and Baldani, 2006; Yang et al., 2009). For instance, promotion of dehydration tolerance is reported in different associated pairs obtained from some $A$. brasilense strains and wheat (Triticum aestivum L.) cultivars (Díaz-Zorita and Fernández-Canigia, 2009).

PGPRs can affect the expression of stress-responsive genes and thus modulate plant responses to stress (Jakab et al., 2005; Gachomo et al., 2014; Barnawal et al., 2017). For example, up-regulation of RD29A gene in Arabidopsis plants inoculated with a PGPR strain (Bradyrhizobium japonicum IRAT FA3) was observed when compared to noninoculated plants (Jakab et al., 2005; Gachomo et al., 2014). DRE/CRT (Dehydration responsive element/C-repeat), a cis-acting element in the promoter region of RD29A (Yamaguchi-Shinozaki and Shinozaki, 2005; Jia et al., 2012; Gachomo et al., 2014) interacts with a series of transcription factors known as DREBs proteins (DREB/CBF; DRE-binding factor/C-repeat binding factor) inducing a set of downstream dehydration responsive genes that improve dehydration tolerance of plants (Baker et al., 1994; Sakuma et al., 2002; Yamaguchi-Shinozaki and Shinozaki, 2005). DREBs genes family which encode DREBs proteins has a main role in the gene expression and regulation of plant's response to dehydration stress (Sakuma et al., 2002; Yamaguchi-Shinozaki and Shinozaki, 2005; Agarwal et al., 2006, 2010). A rapid and transient increase in DREB2 expression causes an increase in the regulatory and/or functional proteins which shows the upstream role of DREB2 in a signaling pathway and improves plant tolerance to 
short-time dehydration. Recently, the effect of some PGPRs such as Arthrobacter protophormiae (SA3), Dietzia natronolimnaea (STR1) and Bacillus subtilis (LDR2) on TaCTR1/TaDREB2 expression (Barnawal et al., 2017) and improvement of wheat tolerance to abiotic stress has been reported but, the effect of A. brasilense on DREB2 expression is not well studied in inoculated wheat (T. aestivum L.) cultivars under dehydration condition.

\section{Materials and methods}

\section{Primary experiment}

A primary experiment (unpublished data) was conducted to choose the most tolerant and sensitive cultivars to drought as well as drought tolerant and sensitive associated pairs among the combination of six wheat cultivars of common wheat (T. aestivum L.) named Roshan, Sardari, Tabasi, Shahpasand, Omid and Shoaleh inoculated with two strains of A. brasilense called Sp7 (as standard strain) and Sp245 (reported to produce significant ABA). The seeds were prepared from Agriculture and Natural Resources Research Center, Isfahan, Iran. The strains of $A$. brasilense strains were obtained from Research Association of AMP Research Unit Microbe-Plant Interactions, Germany.

The growth and biochemical parameters of six wheat cultivars as well as their combinations with two strains of $A$. brasilense ( $\mathrm{Sp} 7$ and $\mathrm{Sp} 245$ ) including roots and shoots dry weight (RDW, SDW), average roots length (ARL), number of main roots (NMR) and the number of root branches (NRB), maximum shoot length (MSL) as well as total protein of roots and shoots (RTP, STP), PAL enzyme activity in roots and shoots (RPAL, SPAL) and activity of TAL enzyme in roots and shoots (RTAL, STAL) were measured. Adopted method of Bradford (1976) was used for protein assay and the method of Beaudoin-Egan and Thorpe (1985) was used for TAL and PAL enzymes activity analysis. The experiment was conducted under drought condition (40\% of field capacity) and compared to $80 \%$ moisture content (data were not shown). Then, the data was ranked for the best and the worst responses to drought (Appendix 1). Afterward, according to the measured parameters, the two cultivars of Roshan and Shahpasand were selected as drought tolerant and sensitive cultivars, respectively. Simultaneously, the associated pairs of cultivar-strain including Roshan-Sp245, Roshan-Sp7, Shahpasand-Sp7 and, Shahpasand-Sp245 were selected as tolerant, moderately tolerant, sensitive and moderately sensitive to drought within all possible combinations of wheat cultivars and A. brasilense strains (Appendix 1). Then, in the second experiment, the selected cultivars and associated pairs were evaluated for DREB2 gene expression under optimum $\left(\psi_{\mathrm{w}}=0 \mathrm{MPa}\right)$ and dehydration $\left(\psi_{\mathrm{w}}=-90 \mathrm{MPa}\right)$ conditions.

\section{Second experiment}

A. brasilense strains were cultured on solid NFB (Nitrogen free basal) medium and incubated at $32^{\circ} \mathrm{C}$. Then the bacteria were cultured in liquid NFB medium enriched with ammonium chloride $\left(0.1 \% \mathrm{w} / \mathrm{v}, 1 \mathrm{~g} \mathrm{~L}^{-1}\right)$ and incubated at $30^{\circ} \mathrm{C}$ for $48 \mathrm{~h}$. Bacterial concentration of $10^{8} \mathrm{CFU} \mathrm{mL} \mathrm{m}^{-1}$ was adjusted by reading optical density at $600 \mathrm{~nm}$ using McFarland table (Krieg and Dobereiner, 1984; McFarland, 1907).

Seeds were sterilized using the method adopted by Sweet and Bolton (1979). Then the sterilized seeds were incubated in sterile distilled water at room temperature for $3 \mathrm{~h}$. Wheat seeds were shaken in high phosphate Nfb (HPNfb) liquid medium enriched with 


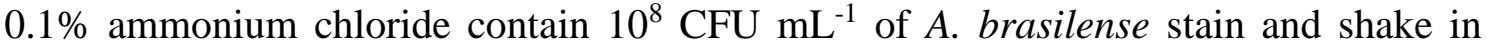
$100 \mathrm{rpm}$ for $3 \mathrm{~h}$ (Shaker Model INFORS AG, BOTTMINGEN) (Okon et al., 1977). Under sterile laminar airflow, the seeds were left to reduce the seeds moisture down to almost $14 \%$.

Sterilized wheat seeds (Shahpasand as sensitive and Roshan as tolerant cultivar) were planted on sterile water agar ( $8 \% \mathrm{w} / \mathrm{v})$ and kept in dark at room temperature for $48 \mathrm{~h}$ for germination. The uniform small seedlings were transferred into test tube $(3 \times 25 \mathrm{~cm})$ containing $40 \mathrm{ml}$ of autoclaved MS medium (30 g sucrose, $7 \mathrm{~g}$ agar, $\mathrm{pH} \sim 6.5$ ) (Murashige and Skoog, 1962) and then located in growth chamber at $22 \pm 2{ }^{\circ} \mathrm{C}, 60 \% \mathrm{RH}$, and photoperiod of $16 \mathrm{~L} / 8 \mathrm{D}\left(210\right.$ photons $\left.\mathrm{m}^{-2} \mathrm{~s}^{-1}\right)$ for 3 more days. Half of the seedlings (5-day-old) were inoculated with specific $A$. brasilense strains.

To inoculate seedling, $\mathrm{Nfb}$ liquid medium enriched with $0.1 \%$ ammonium chloride

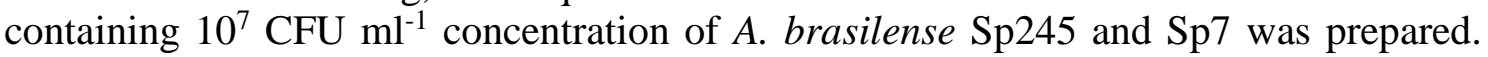
Then under sterile laminar airflow, $1 \mathrm{~mL}$ of $\mathrm{Nfb}$ liquid medium containing Sp245 or Sp7 was added separately into each test tube, containing three 5-day-old seedlings and kept for 2 more days. After 2 days, seedling's roots were checked for the number of bacteria. To do so, the samples of seedling's roots were stained with Iodonitrotetrazolium chloride (INT) dye $(0.25 \% \mathrm{w} / \mathrm{v}$ aqueous solution) and then many cross-sections of root were provided manually (Sriskandarajah et al., 1993). Afterward, the presence of the bacteria within root cross-sections was checked using a light microscope. Meanwhile, the number of bacteria colonies ( $\mathrm{CFU} \mathrm{mL} \mathrm{mL}^{-1}$ ) within the roots was counted. To do so, the root samples were placed in $95 \%(\mathrm{v} / \mathrm{v})$ ethanol for $3 \mathrm{sec}$, then in $0.01 \%(\mathrm{w} / \mathrm{v}) \mathrm{HgCl}_{2}$ for $15 \mathrm{sec}$ and washed several times with sterile water for surface-sterilization (Sriskandarajah et al., 1993). Then, $1 \mathrm{~g}$ of root samples were grounded using $5 \mathrm{~mL}$ of $1 \mathrm{X}$ phosphate buffered saline (PBS) $(\mathrm{pH} \sim 7.4)$ at the sterile condition and serially diluted $\left(10^{-1}\right.$ up to $10^{-9}$ ) (Stets et al., 2015). $5 \mu$ of the final dilution $\left(10^{-9}\right)$ were cultured on solid medium $(8 \% \mathrm{w} / \mathrm{v}$ agar) of HPNfb enriched with ammonium chloride $(0.1 \% \mathrm{w} / \mathrm{w})$ and incubated at $32^{\circ} \mathrm{C}$ for $72 \mathrm{~h}$. At last, the number of colonies formed on the plates was counted and considered as bacterial population in $1 \mathrm{~g}$ of tissue fresh weight. The number of bacteria in root was almost $10^{8} \mathrm{CFU} \mathrm{mL} \mathrm{mL}^{-1}$ for Sp245 and $10^{7}$ CFU $\mathrm{mL}^{-1}$ for Sp7.

One series of 7-day-old seedlings (including non-inoculated and inoculated with A. brasilense strains) were transferred into sterile test tubes $(3 \times 25 \mathrm{~cm})$ which had been saturated with water vapors $\left(\mathrm{RH} \approx 100 \%\right.$ ) at $25^{\circ} \mathrm{C}$ (Thomson, 1872). According to Kelvin equation and after equilibrium of water vapor pressure, this was equal to maximum water potential $\left(\psi_{\mathrm{w}}=0 \mathrm{MPa}\right)$ and was considered as optimum water condition (Butt et al., 2006). The second series of 7-day-old seedlings were transferred into sterile test tubes with $\mathrm{RH}$ equal to $50 \%\left(\psi_{\mathrm{w}}=-90 \mathrm{MPa}\right)$ at $25^{\circ} \mathrm{C}$ as dehydration treatment. After implementation and equilibrium of treatments, plant samples were collected at 0, 120 and 360 minutes. Samples were separated immediately into shoot and root and frozen in liquid nitrogen to store at $-80^{\circ} \mathrm{C}$ for RNA isolation.

\section{Total RNA extraction and quantitative Real-Time PCR (qRT-PCR) analysis}

Total RNA of the shoot and root samples of wheat cultivars were extracted using Irizol kit (Irizol RNA Biotech, Iran) at $4^{\circ} \mathrm{C}$. To remove the possible DNA contamination, the RNA samples was treated with RNase-free DNase I (TaKaRa, Japan). 
About $1 \mu \mathrm{g}$ of total RNA was used to synthesize first-strand cDNA primed with oligo (dT) in a $10 \mu \mathrm{l}$ reaction mix using reverse transcriptase (TaKaRa, Japan) according to the instructions of the manufacturer. QRT-PCR was performed using SYBER-Green dye (TaKaRa, Japan) on real-time PCR machine (Bio-Rad, USA) in triplicate. The ARF (ADP-ribosylation factor) gene (AB050957) was used as the reference gene. The primers $\quad(A R F, \quad \mathrm{~F}$ : 5'-CTGACGCCGAGGATATCCA-3'; R: 5'GCCTTGACCATACCAGTTCCA-3') used for Real-Time PCR analysis were designed using AlleleID, version 7. The amount of transcript accumulated for the DREB2 as the target gene (AY781345) (DREB2, F: 5'-AAGAAAACAGGCGACAAGAT-3'; and R: 5'- GTTGTTGGTTCACTTCTTTC -3') (Egawa et al., 2006) normalized to ARF gene as internal control. The value of DREB2 transcript level was reported relative to the ARF gene $\left(-\Delta \mathrm{C}_{\mathrm{T}}\right)$ which is the negative difference between the threshold cycle $\left(\mathrm{C}_{\mathrm{T}}\right)$ of the target gene and $\mathrm{C}_{\mathrm{T}}$ of the reference gene. Then, the relative expression of DREB 2 in treated conditions (inoculated and/or dehydrated) was calculated using the $2^{-\Delta \Delta C_{T}}$ method relative to control condition (non-inoculation and optimum water condition) (Livak and Schmittgen, 2001). The real-time reaction efficiencies of the target and reference genes ( $\mathrm{E}=0.93$ and 0.96 , respectively) were assumed to be equal, nearly 1 . The PCR cycling conditions including an optimum concentration of primers and cDNA as well as optimum temperature for $A R F$ and $D R E B 2$ genes were set up. So, real-time PCR program was: incubation at $95^{\circ} \mathrm{C}$ for $10 \mathrm{~min}, 95^{\circ} \mathrm{C}$ for $10 \mathrm{~s}, 58^{\circ} \mathrm{C}$ for $25 \mathrm{~s}$ for 40 cycles, $72^{\circ} \mathrm{C}$ for $30 \mathrm{~s}$ and $72^{\circ} \mathrm{C}$ for $7 \mathrm{~min}$.

\section{Statistical analysis}

The primary experiment was conducted in a split plot layout with 3 replicates. The main plot was wheat cultivars, sub-plot was bacterial strains and sub-sub-plot was water condition. The second experiment also carried out as split-plot with 3 replicates. The main plot was sensitivity to drought, inoculation as sub-plot and water condition as subsub-plot.

The ANOVA were obtained using SPSS and the mean values were compared using Duncan's multiple range tests at $95 \%$ confidence interval.

\section{Results}

According to the primary experiment, the associated pairs (wheat cultivarsA. brasilense strains) including tolerant (Roshan-Sp245), moderately tolerant (RoshanSp7), sensitive (Shahpasand-Sp7) and moderately sensitive (Shahpasand-Sp245) to drought were selected (Appendix 1). Physiological responses in drought condition are presented in Tables 1 and 2.

\section{Physiological responses of sensitive and tolerant associated pairs to drought}

Cross-inoculation of Roshan and Shahpasand cultivars with Sp245 or Sp7 strains formed different associated pairs with different physiological responses in optimum water condition $(\mathrm{P} \leq 0.05)$ (Tables 1-4). In optimum water condition, most physiological parameters increased (except for NMR and SPAL) $(\mathrm{P} \leq 0.05)$ when inoculated tolerant cultivar (Roshan- Sp245) compared to non-inoculated one (Roshan). However, in Roshan-Sp7, ARL, RDW and, RTP were the only parameters positively affected by inoculation in optimum water condition (Tables 3 and 4). Although, 
inoculation of the sensitive cultivar (Shahpasand) with Sp7 did not significantly affect any of the physiological parameters at optimum water condition in Shahpasand-Sp245, NRB, SDW, RTP and, RPAL increased due to inoculation $(\mathrm{P} \leq 0.05)($ Tables 1-4). It seems that Sp245 has more homology or compatibility potential to associate with different wheat cultivars than $\mathrm{Sp} 7$.

Table 1. Average values of growth parameters including maximum shoot length (MSL), average root length (ARL), number of main roots (NMR), number of root branches (NRB) and shoot and root dry weight (SDW and RDW, respectively), in non-inoculated cultivars (Roshan and Shahpasand as tolerant and sensitive to drought, respectively) as well as different pairs of wheat cultivars and A. brasilense strains (Sp245 and Sp7) including Roshan-Sp245 (tolerant), Roshan-Sp7 (moderately tolerant), Shahpasand-Sp7 (sensitive), Shahpasand-Sp245 (moderately sensitive) under drought (40\% FC) and optimum water conditions $(80 \%$ FC). Data represent the mean values $\pm S E(n=3)$. Different letters in the mean values indicated the difference between the mean values (Duncan's multiple range tests, $P \leq 0.05)$. MSL and ARL are in $\mathrm{cm} \mathrm{plant}^{-1} ; S D W$ and $R D W$ are in $\mathrm{mg}^{\text {plant }}{ }^{-1} . F C$, stand for moisture content at field capacity

\begin{tabular}{c|c|c|c|c|c|c|c}
\hline FC & Cultivar, Strain & MSL & ARL & NMR & NRB & SDW & RDW \\
\hline $80 \%$ & Roshan cv. & $25.3 \pm 0.2^{\mathrm{b}}$ & $5.7 \pm 0.2^{\mathrm{c}}$ & $5.0 \pm 0.01^{\mathrm{a}}$ & $0.7 \pm 0.6^{\mathrm{c}}$ & $25.0 \pm 0.01^{\mathrm{b}}$ & $9.0 \pm 0.01^{\mathrm{c}}$ \\
& Roshan-Sp245 & $31.2 \pm 0.8^{\mathrm{a}}$ & $10.6 \pm 0.1^{\mathrm{a}}$ & $5.3 \pm 0.6^{\mathrm{a}}$ & $2.3 \pm 0.6^{\mathrm{b}}$ & $29.3 \pm 0.6^{\mathrm{a}}$ & $17.3 \pm 0.6^{\mathrm{a}}$ \\
& Roshan-Sp7 & $26.3 \pm 0.3^{\mathrm{b}}$ & $7.4 \pm 0.2^{\mathrm{b}}$ & $5.0 \pm 0.01^{\mathrm{a}}$ & $1.3 \pm 0.6^{\mathrm{c}}$ & $26.0 \pm 1.0^{\mathrm{b}}$ & $12.7 \pm 0.6^{\mathrm{b}}$ \\
\cline { 2 - 7 } & Shahpasand cv. & $17.8 \pm 0.3^{\mathrm{c}}$ & $7.1 \pm 0.1^{\mathrm{b}}$ & $4.0 \pm 1.0^{\mathrm{b}}$ & $0.0 \pm 0.01^{\mathrm{d}}$ & $20.7 \pm 0.6^{\mathrm{c}}$ & $7.0 \pm 0.01^{\mathrm{c}}$ \\
& Shahpasand-Sp245 & $19.7 \pm 0.6^{\mathrm{c}}$ & $7.6 \pm 0.1^{\mathrm{b}}$ & $4.0 \pm 0.01^{\mathrm{b}}$ & $1.0 \pm 0.01^{\mathrm{c}}$ & $24.0 \pm 1.0^{\mathrm{b}}$ & $7.0 \pm 0.01^{\mathrm{c}}$ \\
& Shahpasand-Sp7 & $18.8 \pm 0.3^{\mathrm{c}}$ & $7.4 \pm 0.1^{\mathrm{b}}$ & $3.7 \pm 0.6^{\mathrm{b}}$ & $0.7 \pm 0.6^{\mathrm{c}}$ & $22.0 \pm 0.01^{\mathrm{c}}$ & $7.0 \pm 0.01^{\mathrm{c}}$ \\
\hline \multirow{2}{*}{$40 \%$} & Roshan cv. & $24.0 \pm 0.2^{\mathrm{b}}$ & $5.4 \pm 0.1^{\mathrm{c}}$ & $5.0 \pm 0.01^{\mathrm{a}}$ & $2.0 \pm 0.01^{\mathrm{b}}$ & $24.0 \pm 0.01^{\mathrm{b}}$ & $8.0 \pm 0.01^{\mathrm{c}}$ \\
& Roshan-Sp245 & $26.4 \pm 0.5^{\mathrm{b}}$ & $7.4 \pm 0.4^{\mathrm{b}}$ & $5.0 \pm 0.01^{\mathrm{a}}$ & $3.7 \pm 0.6^{\mathrm{a}}$ & $27.7 \pm 0.6^{\mathrm{a}}$ & $12.0 \pm 0.01^{\mathrm{b}}$ \\
& Roshan-Sp7 & $24.3 \pm 0.2^{\mathrm{b}}$ & $6.2 \pm 0.1^{\mathrm{b}}$ & $5.0 \pm 0.01^{\mathrm{a}}$ & $2.3 \pm 0.6^{\mathrm{b}}$ & $25.0 \pm 0.01^{\mathrm{b}}$ & $9.0 \pm 0.01^{\mathrm{c}}$ \\
\cline { 2 - 7 } & Shahpasand cv. & $13.8 \pm 0.5^{\mathrm{d}}$ & $5.4 \pm 0.1^{\mathrm{c}}$ & $4.3 \pm 0.6^{\mathrm{b}}$ & $2.0 \pm 0.01^{\mathrm{b}}$ & $16.0 \pm 1.0^{\mathrm{d}}$ & $5.0 \pm 0.01^{\mathrm{d}}$ \\
& Shahpasand-Sp245 & $13.5 \pm 0.5^{\mathrm{d}}$ & $5.4 \pm 0.1^{\mathrm{c}}$ & $4.7 \pm 0.6^{\mathrm{ab}}$ & $1.3 \pm 0.6^{\mathrm{c}}$ & $16.0 \pm 1.0^{\mathrm{d}}$ & $5.0 \pm 0.01^{\mathrm{d}}$ \\
& Shahpasand-Sp7 & $9.4 \pm 0.5^{\mathrm{e}}$ & $3.8 \pm 0.1^{\mathrm{d}}$ & $3.0 \pm 0.01^{\mathrm{c}}$ & $1.7 \pm 0.6^{\mathrm{b}}$ & $11.0 \pm 1.7^{\mathrm{e}}$ & $2.0 \pm 0.01^{\mathrm{e}}$ \\
\hline
\end{tabular}

Table 2. Average values of total protein in shoots (STP) and roots (RTP), activity of PAL enzyme in shoots (SPAL) and roots (RPAL), activity of TAL enzyme in shoot (STAL) and root (RTAL) in non-inoculated cultivars (Roshan and Shahpasand as tolerant and sensitive to drought, respectively) as well as different pairs of wheat cultivars and A. brasilense strains (Sp245 and Sp7) including Roshan-Sp245 (tolerant), Roshan-Sp7 (moderately tolerant), Shahpasand-Sp7 (sensitive), Shahpasand-Sp245 (moderately sensitive) under drought $(40 \%$ FC) and optimum water conditions $(80 \%$ FC). Data represent the mean values $\pm S E$ $(n=3)$. Different letters in the mean values indicated the difference between the means (Duncan's multiple range tests, $P \leq 0.05$ ). STP and RTP are is $m g g F W^{1}$; SPAL, RPAL, STAL and RTAL are in U mg protein ${ }^{-1}$. FC, stand for moisture content at field capacity

\begin{tabular}{c|c|c|c|c|c|c|c}
\hline FC & Cultivar, Strain & STP & RTP & SPAL & RPAL & STAL & RTAL \\
\hline $80 \%$ & Roshan cv. & $44.0 \pm 0.5^{\mathrm{b}}$ & $37.4 \pm 1.1^{\mathrm{c}}$ & $17.4 \pm 0.01^{\mathrm{c}}$ & $11.7 \pm 0.2^{\mathrm{b}}$ & $20.7 \pm 0.1^{\mathrm{c}}$ & $15.3 \pm 0.01^{\mathrm{d}}$ \\
& Roshan-Sp245 & $86.2 \pm 0.5^{\mathrm{a}}$ & $79.3 \pm 1.0^{\mathrm{a}}$ & $20.6 \pm 0.1^{\mathrm{b}}$ & $14.5 \pm 0.3^{\mathrm{a}}$ & $23.6 \pm 0.1^{\mathrm{b}}$ & $19.3 \pm 0.6^{\mathrm{c}}$ \\
& Roshan-Sp7 & $45.0 \pm 0.2^{\mathrm{b}}$ & $47.3 \pm 0.7^{\mathrm{bc}}$ & $18.0 \pm 0.1^{\mathrm{c}}$ & $12.4 \pm 0.1^{\mathrm{b}}$ & $21.9 \pm 0.1^{\mathrm{c}}$ & $16.0 \pm 0.4^{\mathrm{d}}$ \\
\cline { 2 - 7 } & Shahpasand cv. & $32.7 \pm 0.3^{\mathrm{c}}$ & $26.0 \pm 1.3^{\mathrm{d}}$ & $10.8 \pm 0.1^{\mathrm{d}}$ & $9.0 \pm 0.01^{\mathrm{c}}$ & $19.4 \pm 0.1^{\mathrm{c}}$ & $12.1 \pm 0.3^{\mathrm{e}}$ \\
& Shahpasand-Sp245 & $37.6 \pm 0.5^{\mathrm{c}}$ & $35.0 \pm 1.5^{\mathrm{c}}$ & $12.3 \pm 0.2^{\mathrm{d}}$ & $11.5 \pm 0.3^{\mathrm{b}}$ & $21.0 \pm 0.1^{\mathrm{c}}$ & $13.3 \pm 0.3^{\mathrm{e}}$ \\
& Shahpasand-Sp7 & $36.1 \pm 1.2^{\mathrm{c}}$ & $29.2 \pm 1.2^{\mathrm{d}}$ & $11.1 \pm 0.3^{\mathrm{d}}$ & $9.9 \pm 0.1^{\mathrm{c}}$ & $19.7 \pm 0.1^{\mathrm{c}}$ & $12.5 \pm 0.3^{\mathrm{e}}$ \\
\hline \multirow{2}{*}{$40 \%$} & Roshan cv. & $50.9 \pm 0.9^{\mathrm{b}}$ & $56.1 \pm 4.6^{\mathrm{b}}$ & $23.6 \pm 0.6^{\mathrm{a}}$ & $12.9 \pm 0.1^{\mathrm{b}}$ & $24.8 \pm 0.2^{\mathrm{b}}$ & $18.3 \pm 0.6^{\mathrm{c}}$ \\
& Roshan-Sp245 & $55.7 \pm 0.7^{\mathrm{b}}$ & $79.3 \pm 0.0^{\mathrm{a}}$ & $26.2 \pm 0.1^{\mathrm{a}}$ & $15.4 \pm 0.01^{\mathrm{a}}$ & $30.3 \pm 0.1^{\mathrm{a}}$ & $25.0 \pm 0.7^{\mathrm{a}}$ \\
& Roshan-Sp7 & $52.0 \pm 0.4^{\mathrm{b}}$ & $70.3 \pm 1.8^{\mathrm{a}}$ & $24.2 \pm 0.1^{\mathrm{a}}$ & $13.5 \pm 0.3^{\mathrm{b}}$ & $26.0 \pm 0.1^{\mathrm{b}}$ & $22.3 \pm 1.2^{\mathrm{b}}$ \\
\cline { 2 - 7 } & Shahpasand cv. & $30.3 \pm 0.3^{\mathrm{c}}$ & $21.5 \pm 1.1^{\mathrm{d}}$ & $9.7 \pm 0.1^{\mathrm{d}}$ & $8.1 \pm 0.1^{\mathrm{ce}}$ & $17.9 \pm 0.1^{\mathrm{c}}$ & $10.2 \pm 0.3^{\mathrm{e}}$ \\
& Shahpasand-Sp245 & $32.2 \pm 0.7^{\mathrm{c}}$ & $21.2 \pm 0.2^{\mathrm{d}}$ & $8.6 \pm 0.1^{\mathrm{d}}$ & $7.2 \pm 0.3^{\mathrm{e}}$ & $16.6 \pm 0.1^{\mathrm{c}}$ & $9.20 \pm 0.3^{\mathrm{ef}}$ \\
& Shahpasand-Sp7 & $32.3 \pm 0.3^{\mathrm{c}}$ & $19.4 \pm 0.9^{\mathrm{d}}$ & $8.3 \pm 0.01^{\mathrm{d}}$ & $7.0 \pm 0.2^{\mathrm{e}}$ & $15.2 \pm 0.1^{\mathrm{d}}$ & $6.5 \pm 0.6^{\mathrm{f}}$ \\
\hline
\end{tabular}


Table 3. Based on the values of Table 1, the percent of changes in each parameter of different associated pairs due to inoculation and/or drought conditions. Optimum water condition defined as $80 \%$ FC and drought as 40\% FC (moisture content at field capacity). Abbreviation: maximum shoot length (MSL), average roots length (ARL), number of main roots (NMR), number of roots branches (NRB) and shoots and roots dry weight (SDW and $R D W$, respectively)

\begin{tabular}{|c|c|c|c|c|c|}
\hline $\begin{array}{c}\text { Growth } \\
\text { parameters }\end{array}$ & $\begin{array}{c}\text { Sensitivity of associated } \\
\text { pairs } \\
\text { to drought }\end{array}$ & $\begin{array}{c}\text { At } 80 \% \text { FC } \\
\text { Inoculated } \\
\text { versus } \\
\text { Non-inoculated }\end{array}$ & $\begin{array}{c}\begin{array}{c}\text { For non- } \\
\text { inoculated }\end{array} \\
40 \% \\
\text { versus } \\
80 \% \\
\end{array}$ & $\begin{array}{c}\begin{array}{c}\text { For } \\
\text { Inoculated }\end{array} \\
\mathbf{4 0 \%} \\
\text { versus } \\
\mathbf{8 0 \%} \\
\end{array}$ & $\begin{array}{c}\text { Inoculated } 40 \% \\
\text { versus } \\
\text { Non-inoculated } 80 \%\end{array}$ \\
\hline & & $\begin{array}{c}\text { Inoculation } \\
\text { effect }\end{array}$ & $\begin{array}{c}\text { Drought } \\
\text { effect }\end{array}$ & $\begin{array}{c}\text { Drought effect } \\
\text { under inoculation }\end{array}$ & $\begin{array}{c}\text { Dual } \\
\text { effect }\end{array}$ \\
\hline MSL & $\begin{array}{c}\text { Tolerant } \\
\text { Moderately tolerant } \\
\text { Sensitive } \\
\text { Moderately sensitive } \\
\end{array}$ & $\begin{array}{c}23 \\
0 \\
0 \\
0 \\
\end{array}$ & $\begin{array}{c}0 \\
- \\
-25 \\
- \\
\end{array}$ & $\begin{array}{c}-15 \\
0 \\
-50 \\
-31 \\
\end{array}$ & $\begin{array}{c}0 \\
0 \\
-47 \\
-24 \\
\end{array}$ \\
\hline ARL & $\begin{array}{c}\text { Tolerant } \\
\text { Moderately tolerant } \\
\text { Sensitive } \\
\text { Moderate sensitive }\end{array}$ & $\begin{array}{c}85 \\
30 \\
0 \\
0\end{array}$ & $\begin{array}{c}0 \\
- \\
-25 \\
-\end{array}$ & $\begin{array}{c}-30 \\
0 \\
-50 \\
-29\end{array}$ & $\begin{array}{c}29 \\
9 \\
-46 \\
-24\end{array}$ \\
\hline NMR & $\begin{array}{c}\text { Tolerant } \\
\text { Moderately tolerant } \\
\text { Sensitive } \\
\text { Moderately sensitive } \\
\end{array}$ & $\begin{array}{l}0 \\
0 \\
0 \\
0\end{array}$ & $\begin{array}{l}0 \\
- \\
0 \\
-\end{array}$ & $\begin{array}{c}0 \\
0 \\
-18 \\
0 \\
\end{array}$ & $\begin{array}{c}0 \\
0 \\
-25 \\
0\end{array}$ \\
\hline NRB & $\begin{array}{c}\text { Tolerant } \\
\text { Moderately tolerant } \\
\text { Sensitive } \\
\text { Moderately sensitive } \\
\end{array}$ & $\begin{array}{c}250 \\
0 \\
0 \\
1 * \\
\end{array}$ & $\begin{array}{c}198 \\
- \\
2 * \\
- \\
\end{array}$ & $\begin{array}{c}61 \\
77 \\
143 \\
0 \\
\end{array}$ & $\begin{array}{c}448 \\
229 \\
1.67^{*} \\
1.3^{*} \\
\end{array}$ \\
\hline SDW & $\begin{array}{c}\text { Tolerant } \\
\text { Moderately tolerant } \\
\text { Sensitive } \\
\text { Moderately sensitive }\end{array}$ & $\begin{array}{c}16 \\
0 \\
0 \\
16\end{array}$ & $\begin{array}{c}0 \\
- \\
-25 \\
- \\
\end{array}$ & $\begin{array}{c}0 \\
0 \\
-50 \\
-33 \\
\end{array}$ & $\begin{array}{c}12 \\
0 \\
-47 \\
-23 \\
\end{array}$ \\
\hline RDW & $\begin{array}{c}\text { Tolerant } \\
\text { Moderately tolerant } \\
\text { Sensitive } \\
\text { Moderately sensitive }\end{array}$ & $\begin{array}{c}89 \\
41 \\
0 \\
0\end{array}$ & $\begin{array}{c}0 \\
- \\
-25 \\
-\end{array}$ & $\begin{array}{l}-31 \\
-29 \\
-71 \\
-28\end{array}$ & $\begin{array}{c}33 \\
0 \\
-71 \\
-28\end{array}$ \\
\hline
\end{tabular}

*: Indication increased NRB from 0

The selected cultivars or associated pairs showed different physiological responses to drought $(\mathrm{P} \leq 0.05)$ (Tables 1-4). At drought condition, MSL, ARL and, RDW in Roshan $\mathrm{cv}$. (drought tolerant) did not decrease as compared to optimum water condition, but these parameters were decreased in Shahpasand cv. (Tables 1 and 3). In drought condition, RDW decreased in all pairs with the highest in Shahpasand-Sp7 and the lowest in Roshan-Sp245. SDW of Roshan cv. in non-inoculated and inoculated status did not change in drought condition but SDW of Shahpasand cv. and inoculated Shahpasand cv. with any of two strains were decreased. The drought condition had a negative effect on NMR of Shahpasand with Sp7. However, NRB increased in all cultivars and pairs except for Shahpasand-Sp245 under drought condition $(\mathrm{P} \leq 0.05)$ (Tables 1-4).

STAL and RTAL were increased in inoculated Roshan cv. with Sp245 or Sp7 but they decreased in Shahpasand cv. with both strains. In contrast, SPAL in Roshan cv. and Roshan inoculated with Sp245 or Sp7 were increased in drought condition but they did 
not change in Shahpasand cv. or Shahpasand with Sp7 or Sp245. STP did not change due to drought in all cultivars and pairs except for Roshan-Sp245. Meanwhile, RTP did not change in Roshan-Sp245 and Shahpasand-Sp7 but it was increased in Roshan-Sp7 and decreased in Shahpasand-Sp245 under drought condition (Tables 2 and 4).

Table 4. Based on the values of Table 2, the percent of changes in each parameter of different associated pairs due to inoculation and/or drought conditions. Optimum water condition defined as $80 \%$ FC and drought as 40\% FC (moisture content at field capacity). Abbreviation: total protein of roots (RTP) and shoots (STP, the activity of PAL enzyme in shoots (SPAL) and roots (RPAL), the activity of TAL enzyme in shoots (STAL) and roots (RTAL)

\begin{tabular}{|c|c|c|c|c|c|}
\hline $\begin{array}{c}\text { Growth } \\
\text { parameters }\end{array}$ & $\begin{array}{l}\text { Sensitivity of } \\
\text { associated pairs } \\
\text { to drought }\end{array}$ & \begin{tabular}{|c|} 
At $80 \%$ FC \\
Inoculated \\
versus \\
Non-inoculated
\end{tabular} & $\begin{array}{c}\begin{array}{c}\text { For non- } \\
\text { inoculated }\end{array} \\
40 \% \\
\text { versus } \\
\mathbf{8 0 \%}\end{array}$ & $\begin{array}{c}\begin{array}{c}\text { For } \\
\text { Inoculated }\end{array} \\
\mathbf{4 0 \%} \\
\text { versus } \\
\mathbf{8 0 \%}\end{array}$ & $\begin{array}{c}\text { Inoculated } 40 \% \\
\text { versus } \\
\text { Non-inoculated } \\
\mathbf{8 0 \%}\end{array}$ \\
\hline & & $\begin{array}{c}\text { Inoculation } \\
\text { effect }\end{array}$ & $\begin{array}{c}\text { Drought } \\
\text { effect }\end{array}$ & $\begin{array}{c}\text { Drought effect } \\
\text { under inoculation }\end{array}$ & $\begin{array}{c}\text { Dual } \\
\text { effect }\end{array}$ \\
\hline STP & $\begin{array}{c}\text { Tolerant } \\
\text { Moderately tolerant } \\
\text { Sensitive } \\
\text { Moderately sensitive }\end{array}$ & $\begin{array}{c}96 \\
0 \\
0 \\
0 \\
\end{array}$ & $\begin{array}{l}0 \\
- \\
0 \\
-\end{array}$ & $\begin{array}{c}-35 \\
0 \\
0 \\
0\end{array}$ & $\begin{array}{l}0 \\
0 \\
0 \\
0\end{array}$ \\
\hline RTP & $\begin{array}{c}\text { Tolerant } \\
\text { Moderately tolerant } \\
\text { Sensitive } \\
\text { Moderately sensitive }\end{array}$ & $\begin{array}{c}110 \\
26 \\
0 \\
35 \\
\end{array}$ & $\begin{array}{c}50 \\
- \\
0 \\
-\end{array}$ & $\begin{array}{c}0 \\
49 \\
0 \\
-39\end{array}$ & $\begin{array}{c}112 \\
88 \\
0 \\
0 \\
\end{array}$ \\
\hline SPAL & $\begin{array}{c}\text { Tolerant } \\
\text { Moderately tolerant } \\
\text { Sensitive } \\
\text { Moderately sensitive }\end{array}$ & $\begin{array}{l}0 \\
0 \\
0 \\
0 \\
\end{array}$ & $\begin{array}{c}36 \\
- \\
0 \\
- \\
\end{array}$ & $\begin{array}{c}27 \\
34 \\
0 \\
0 \\
\end{array}$ & $\begin{array}{c}50 \\
39 \\
0 \\
0 \\
\end{array}$ \\
\hline RPAL & $\begin{array}{c}\text { Tolerant } \\
\text { Moderately tolerant } \\
\text { Sensitive } \\
\text { Moderately sensitive }\end{array}$ & $\begin{array}{c}24 \\
0 \\
0 \\
28\end{array}$ & $\begin{array}{l}0 \\
- \\
0 \\
-\end{array}$ & $\begin{array}{c}0 \\
0 \\
-29 \\
-37 \\
\end{array}$ & $\begin{array}{c}31 \\
0 \\
-29 \\
-20 \\
\end{array}$ \\
\hline STAL & $\begin{array}{c}\text { Tolerant } \\
\text { Moderately tolerant } \\
\text { Sensitive } \\
\text { Moderately sensitive }\end{array}$ & $\begin{array}{c}14 \\
0 \\
0 \\
0 \\
\end{array}$ & $\begin{array}{c}20 \\
- \\
0 \\
- \\
\end{array}$ & $\begin{array}{r}29 \\
19 \\
-23 \\
-37 \\
\end{array}$ & $\begin{array}{c}47 \\
26 \\
-22 \\
0 \\
\end{array}$ \\
\hline RTAL & $\begin{array}{c}\text { Tolerant } \\
\text { Moderately tolerant } \\
\text { Sensitive } \\
\text { Moderately sensitive }\end{array}$ & $\begin{array}{c}27 \\
0 \\
0 \\
0\end{array}$ & $\begin{array}{c}20 \\
- \\
0 \\
-\end{array}$ & $\begin{array}{l}29 \\
39 \\
-48 \\
-31\end{array}$ & $\begin{array}{c}37 \\
46 \\
-47 \\
-24\end{array}$ \\
\hline
\end{tabular}

The dual effects of inoculation and drought were evaluated by comparing an inoculated cultivar which was grown under drought condition with the respective noninoculated cultivar at optimum water condition (named control). When inoculated Roshan cv. with Sp245 or Sp7 under drought condition were compared with control, ARL, NRB, RTP, SPAL, RTAL and, STAL were higher in inoculated one. In contrast, RDW, SDW, ARL, MSL, RPAL, RTAL in Shahpasand cv. with Sp7 or Sp245 under drought condition were lower than control. Simultaneously, MSL, NMR and STP in inoculated Roshan cv. and RTP, STP and, SPAL of inoculated Shahpasand cv. did not show any changes. However, NRB increased in inoculated Shahpasand cv. as compared to control (Tables 1-4). 
In brief, the most beneficial changes in the physiological parameters were observed in the tolerant pair (Roshan-Sp245) followed by moderately tolerant (Roshan-Sp7), moderately sensitive (Shahpasand- Sp245) and sensitive pairs (Shahpasand-Sp7) at either drought or optimum water conditions (Tables 1-4).

\section{DREB2 expression in selected pairs under different water potentials}

At optimum water potential $\left(\psi_{\mathrm{w}}=0 \mathrm{MPa}\right), D R E B 2$ expression in the roots of inoculated seedlings (Tables 5, 6) was less relative to non-inoculated cultivars (ranged from 0.05 up to 0.57 - fold for tolerant to sensitive pairs $(\mathrm{P} \leq 0.05)$ ). However, their expressions were similar at 0,120 and $360 \mathrm{~min}$ after dehydration imposed (Table 5). $D R E B 2$ in the shoots of all selected pairs were up-regulated as compared to noninoculated cultivars. DREB2 up-regulation was more in the shoots of tolerant and moderately tolerant pairs than the sensitive and moderately sensitive pairs $(\mathrm{P} \leq 0.05)$ (Table 6). Therefore, at optimum water condition, DREB2 down-regulated in the roots (Table 5) but up-regulated in the shoots of tolerant and sensitive pairs relative to noninoculated cultivars.

Table 5. DREB2 expression (fold) in different times (0,120 and $360 \mathrm{~min})$ after dehydration $(\psi w=-90 \mathrm{MPa})$ imposed in the roots of different associated pairs of wheat cultivars and A. brasilense strains (Sp245 and Sp7) as responded to drought including: tolerant (RoshanSp245), moderately tolerant (Roshan-Sp7), sensitive (Shahpasand-Sp7), moderately sensitive (Shahpasand-Sp245). The result was compared to optimum water condition ( $\psi w=0 \mathrm{MPa})$. Data represent the mean values $\pm S E(n=3)$. Different letters in the mean values indicated the difference between the means (Duncan's multiple range tests, $P \leq 0.05$ )

\begin{tabular}{|c|c|c|c|c|c|}
\hline \multirow[b]{2}{*}{$\begin{array}{l}\text { Time } \\
(\min )\end{array}$} & \multirow[b]{2}{*}{$\Psi_{\mathbf{w}}(\mathbf{M P a})$} & \multirow[b]{2}{*}{$\begin{array}{l}\text { Sensitivity of } \\
\text { associated pairs } \\
\text { to drought }\end{array}$} & \multirow{2}{*}{$\begin{array}{c}\text { Non-inoculated } \\
\text { Dehydration } \\
\text { versus } \\
\text { optimum }\end{array}$} & \multirow{2}{*}{$\begin{array}{c}\text { Inoculated } \\
\text { Dehydration } \\
\text { versus } \\
\text { optimum }\end{array}$} & \multirow[b]{2}{*}{$\begin{array}{c}\text { Inoculated } \\
\text { versus } \\
\text { Non-inoculated }\end{array}$} \\
\hline & & & & & \\
\hline 0 & $\begin{array}{c}0 \\
\text { (optimum) }\end{array}$ & $\begin{array}{c}\text { Tolerant } \\
\text { Moderately tolerant } \\
\text { Sensitive } \\
\text { Moderately sensitive }\end{array}$ & $\begin{array}{l}- \\
- \\
- \\
-\end{array}$ & $\begin{array}{l}- \\
- \\
- \\
-\end{array}$ & $\begin{array}{l}0.06 \pm 0.02^{\mathrm{c}} \\
0.05 \pm 0.01^{\mathrm{c}} \\
0.15 \pm 0.04^{\mathrm{c}} \\
0.15 \pm 0.02^{\mathrm{c}}\end{array}$ \\
\hline \multirow[t]{2}{*}{120} & 0 & $\begin{array}{c}\text { Tolerant } \\
\text { Moderately tolerant } \\
\text { Sensitive } \\
\text { Moderately sensitive }\end{array}$ & $\begin{array}{l}- \\
- \\
- \\
-\end{array}$ & $\begin{array}{l}- \\
- \\
- \\
-\end{array}$ & $\begin{array}{l}0.07 \pm 0.02^{\mathrm{c}} \\
0.06 \pm 0.02^{\mathrm{c}} \\
0.14 \pm 0.02^{\mathrm{c}} \\
0.16 \pm 0.01^{\mathrm{c}}\end{array}$ \\
\hline & $\begin{array}{c}-90 \\
\text { (dehydrated) }\end{array}$ & $\begin{array}{c}\text { Tolerant } \\
\text { moderately tolerant } \\
\text { Sensitive } \\
\text { Moderately sensitive }\end{array}$ & $\begin{array}{c}2.65 \pm 0.70^{\mathrm{a}} \\
- \\
1.60 \pm 0.02^{\mathrm{b}} \\
-\end{array}$ & $\begin{array}{c}777.8 \pm 100.76^{\mathrm{a}} \\
710.8 \pm 84.44^{\mathrm{a}} \\
20.9 \pm 1.16^{\mathrm{b}} \\
19.1 \pm 2.72^{\mathrm{b}}\end{array}$ & $\begin{array}{c}19.20 \pm 4.83^{\mathrm{a}} \\
17.19 \pm 2.12^{\mathrm{b}} \\
1.87 \pm 0.23^{\mathrm{c}} \\
1.86 \pm 0.42^{\mathrm{c}}\end{array}$ \\
\hline \multirow[t]{2}{*}{360} & 0 & $\begin{array}{c}\text { Tolerant } \\
\text { Moderately tolerant } \\
\text { Sensitive } \\
\text { moderately sensitive }\end{array}$ & $\begin{array}{l}- \\
- \\
- \\
-\end{array}$ & $\begin{array}{l}- \\
- \\
- \\
-\end{array}$ & $\begin{array}{l}0.07 \pm 0.01^{\mathrm{c}} \\
0.06 \pm 0.02^{\mathrm{c}} \\
0.20 \pm 0.52^{\mathrm{c}} \\
0.20 \pm 0.11^{\mathrm{c}}\end{array}$ \\
\hline & -90 & $\begin{array}{c}\text { Tolerant } \\
\text { Moderately tolerant } \\
\text { Sensitive } \\
\text { Moderately sensitive }\end{array}$ & $\begin{array}{c}0.51 \pm 0.14^{\mathrm{c}} \\
- \\
1.93 \pm 0.48^{\mathrm{b}} \\
-\end{array}$ & $\begin{array}{l}3.6 \pm 0.68^{\mathrm{b}} \\
3.4 \pm 0.43^{\mathrm{b}} \\
5.1 \pm 0.56^{\mathrm{b}} \\
5.5 \pm 5.06^{\mathrm{b}}\end{array}$ & $\begin{array}{l}0.51 \pm 0.06^{\mathrm{c}} \\
0.43 \pm 0.10^{\mathrm{c}} \\
0.51 \pm 0.09^{\mathrm{c}} \\
0.57 \pm 0.02^{\mathrm{c}}\end{array}$ \\
\hline
\end{tabular}

Dehydration had a significant effect on DREB2 expression of roots and shoots in inoculated and non-inoculated cultivars $(\mathrm{P} \leq 0.05)$ (Tables 5, 6). The result showed that in the tolerant cultivar (Roshan cv.) at $120 \mathrm{~min}$ after dehydration, DREB2 $\left(2^{-\Delta \Delta \mathrm{C}} \mathrm{T}\right)$ upregulated 2.7 and 3.4- fold in the roots and shoots, respectively as compared to optimum 
water condition. In the sensitive cultivar (Shahpasand cv.), DREB2 did not change in the roots or shoots due to dehydration $(\mathrm{P} \leq 0.05)$ (Table 5). At $360 \mathrm{~min}$, DREB2 expression acts vice versa under dehydration, means, down-regulated in the roots of Roshan (0.5fold) and up-regulated (2.6- fold) in the shoots, but in Shahpasand, DREB2 up-regulated in the roots (1.9- fold) and down-regulated in the shoots (0.6- fold).

The change in DREB2 expression due to dehydration in non-inoculated cultivars, and selected association pairs (the inoculated cultivars) had different sensitivity to drought (Table 5). For example, at 120 min after dehydration, DREB2 up-regulated in the roots of tolerant, moderately tolerant, sensitive and moderately sensitive pairs (778, 711, 21 and 19- fold, respectively) while, DREB2 up-regulated (1-fold) in the shoots of tolerant and moderately tolerant pairs and down-regulated (almost 0.4 - fold) in the sensitive and moderately sensitive pairs (Tables 5, 6). However, at $360 \mathrm{~min}$, DREB2 up-regulation in the roots of all pairs were so less (ranged from 3.4 to 5.5- fold). In contrast, DREB2 down-regulated due to dehydration in the shoots of the tolerant, moderately tolerant (0.2- fold), sensitive and moderately sensitive pairs (0.4- fold) (Tables 5, 6).

Table 6. DREB2 expression (fold) in different times (0,120 and 360 min) after dehydration $\left(\psi_{w}=-90 \mathrm{MPa}\right)$ imposed in the shoots of different associated pairs of wheat cultivars and A. brasilense strains (Sp245 and Sp7) as responded to drought including: tolerant (RoshanSp245), moderately tolerant (Roshan-Sp7), sensitive (Shahpasand-Sp7), moderately sensitive (Shahpasand-Sp245). The result was compared to optimum water condition $\left(\psi_{w}=0 \mathrm{MPa}\right)$. Data represent the mean values $\pm S E(n=3)$. Different letters in the mean values indicated the difference between the means (Duncan's multiple range tests, $P \leq 0.05$ )

\begin{tabular}{|c|c|c|c|c|c|}
\hline \multirow[b]{2}{*}{$\begin{array}{l}\text { Time } \\
\text { (min) }\end{array}$} & \multirow[b]{2}{*}{$\Psi \mathbf{w}(\mathrm{MPa})$} & \multirow[b]{2}{*}{$\begin{array}{l}\text { Sensitivity of } \\
\text { associated pairs } \\
\text { to drought }\end{array}$} & Non-inoculated & Inoculated & \multirow[b]{2}{*}{$\begin{array}{c}\text { Inoculated } \\
\text { versus } \\
\text { Non-inoculated }\end{array}$} \\
\hline & & & $\begin{array}{c}\text { Dehydration } \\
\text { versus } \\
\text { optimum } \\
\end{array}$ & $\begin{array}{c}\text { Dehydration } \\
\text { versus } \\
\text { optimum } \\
\end{array}$ & \\
\hline \multirow[t]{4}{*}{0} & 0 & Tolerant & - & - & $3.43 \pm 0.47^{\mathrm{a}}$ \\
\hline & (control) & Moderately tolerant & - & - & $3.27 \pm 0.70^{\mathrm{a}}$ \\
\hline & & Sensitive & - & - & $1.39 \pm 0.32^{\mathrm{b}}$ \\
\hline & & Moderately sensitive & - & - & $1.40 \pm 0.12^{\mathrm{b}}$ \\
\hline \multirow[t]{8}{*}{120} & 0 & Tolerant & - & - & $3.35 \pm 0.64^{\mathrm{a}}$ \\
\hline & & Moderately tolerant & - & - & $3.32 \pm 0.19^{\mathrm{a}}$ \\
\hline & & Sensitive & - & - & $1.27 \pm 0.01^{\mathrm{b}}$ \\
\hline & & Moderately sensitive & - & - & $1.28 \pm 0.03^{\mathrm{b}}$ \\
\hline & -90 & Tolerant & $3.35 \pm 0.33^{\mathrm{a}}$ & $1.22 \pm 0.29^{\mathrm{a}}$ & $1.22 \pm 0.32^{\mathrm{b}}$ \\
\hline & (dehydrated) & moderately tolerant & - & $1.12 \pm 0.17^{\mathrm{a}}$ & $1.11 \pm 0.19^{\mathrm{b}}$ \\
\hline & & Sensitive & $1.02 \pm 0.31^{\mathrm{b}}$ & $0.38 \pm 0.14^{\mathrm{b}}$ & $0.48 \pm 0.18^{\mathrm{d}}$ \\
\hline & & Moderately sensitive & - & $0.40 \pm 0.09^{\mathrm{b}}$ & $0.50 \pm 0.06^{\mathrm{d}}$ \\
\hline \multirow[t]{8}{*}{360} & 0 & Tolerant & - & - & $3.29 \pm 1.21^{\mathrm{a}}$ \\
\hline & & Moderately tolerant & - & - & $3.08 \pm 0.85^{\mathrm{a}}$ \\
\hline & & Sensitive & - & - & $1.17 \pm 0.02^{\mathrm{b}}$ \\
\hline & & moderately sensitive & - & - & $1.18 \pm 0.34^{\mathrm{b}}$ \\
\hline & -90 & Tolerant & $2.64 \pm 0.47^{\mathrm{a}}$ & $0.16 \pm 0.04^{\mathrm{c}}$ & $0.20 \pm 0.05^{\mathrm{e}}$ \\
\hline & & Moderately tolerant & - & $0.16 \pm 0.08^{c}$ & $0.19 \pm 0.05^{\mathrm{e}}$ \\
\hline & & Sensitive & $0.59 \pm 0.09^{b}$ & $0.43 \pm 0.14^{\mathrm{b}}$ & $0.85 \pm 0.18^{c}$ \\
\hline & & Moderately sensitive & - & $0.45 \pm 0.09^{\mathrm{b}}$ & $0.90 \pm 0.26^{\mathrm{c}}$ \\
\hline
\end{tabular}

To evaluate dual effects of inoculation and dehydration in each pair, the inoculated seedlings which exposed to dehydration condition were compared to the respective noninoculated seedlings grown at optimum water condition. The result showed that at 120 min after dehydration, transcripts level of DREB2 $(-\triangle \mathrm{CT})$ in the tolerant and moderately 
tolerant pairs was more than tolerant cultivar grown at optimum water condition (11.4- fold for roots and 2.3- fold for shoots). Meanwhile, in dehydrated roots of sensitive and moderately sensitive pairs, transcripts level of $D R E B 2$ was more than noninoculated and non-dehydrated roots (1.5- fold) but in their shoot DREB2 was less than non-inoculated ones at optimum water potential (2.6 and 2.4- fold, respectively) $(\mathrm{P} \leq 0.05)(\mathrm{t}=120)$.

In the last measurement after dehydration $(360 \mathrm{~min})$, the transcripts level of DREB2 in the tolerant and moderately tolerant pairs decreased (almost 5 and 6-fold for roots and almost 1-fold for shoot, respectively) as compared to non-inoculated cultivars grown under optimum water potential while in the sensitive and moderately sensitive pairs, there was no change in the roots but it was lower in the shoots (3.7 and 3.4-fold, respectively) $(\mathrm{P} \leq 0.05)$.

\section{Discussion}

The primary experiment was necessary to find out the most sensitive and tolerant cultivars to drought among the usual cultivated wheat cultivars (Roshan, Sardari, Tabasi, Shahpasand, Omid and Shoaleh). Then finding the compatible (or homologous) and incompatible (heterogonous) pairs among association pair of these six cultivars with two strains of A. brasilense (Sp7 and Sp245) under drought condition (Baldani et al., 1983; Jain and Patriquin, 1984; Amooaghaie et al., 2004). This is important due to the complexity of interaction between bacteria and host plant which can affect the successful infection, the establishment of bacterial in host plant and the following appropriate physiological responses (Jain and Patriquin, 1984; Sumner, 1990; Baldani et al., 1986, 1987). According to the physiological parameters such as growth and defensive parameters as well as total protein content, the two cultivars of Roshan and Shahpasand were determined as the most tolerant and sensitive cultivars to drought, respectively (Baldani et al., 1983; Estabrook and Sengupta-Gopalan, 1991; Vasse et al., 1993; Santos et al., 2001). Simultaneously, the best partner of wheat cv. and A. brasilense strains as tolerant pair to drought was Roshan-Sp245 (homologous) and the worst as the most sensitive pair was Shahpasand-Sp7 (heterologous). Also, other pairs from cross-inoculation of Roshan and Shahpasand cultivars with Sp245 and Sp7 strains indicated that Roshan-Sp7 as moderately tolerant and Shahpasand-Sp245 as moderately sensitive pairs.

\section{Physiological responses of the selected pairs to drought}

Cross-inoculation of Roshan and Shahpasand cultivars (as tolerant and sensitive to drought, respectively) with A. brasilense Sp245 and Sp7 strains make different associated pairs with different physiological responses in drought and optimum water conditions. Inoculation of Roshan cv. with A. brasilense Sp245 improved the growth of wheat seedlings except for NMR and SPAL. Similar results have been reported that Sp245 caused higher shoot length, root length and the number of roots (Baldani et al., 1987; Hamdia et al., 2004; Akbari et al., 2007; Cohen et al., 2008; Spaepen et al., 2009). Also, in the moderately tolerant (Roshan-Sp7) pair, ARL, RDW and RTP increased due to inoculation at optimum water condition (Tables 3 and 4). The positive effect of A. brasilense to promote wheat growth could be partly related to the production of more roots and consequently uptake more water and nutrient from the soil (Casimiro et al., 2003; Correa-Aragunde et al., 2004). However, inoculation of Shahpasand cv. (sensitive 
to drought) with $\mathrm{Sp} 7$ (sensitive association pair) did not show any positive effect on the growth parameters but in Shahpasand-Sp245 (moderately sensitive), NRB, SDW, RTP and, RPAL increased due to inoculation. Regarding to previous works, it can be concluded that any strain of bacteria should be homologous to specific wheat cultivar to expect stimulation effect on growth parameter (Baldani et al., 1983; Amooaghaie et al., 2004). In this experiment, not only Sp245 was in favor of tolerant cultivar, but also did a good job with Shahpasand cultivar. In contrast, Sp7 act in favor of Roshan cv. but has no effect on Shahpasand cultivar.

The effect of any bacterial strain on growth and development of wheat cultivars is neither the same nor ended to the growth parameters because in some cases there are no changes in growth indexes but biochemical parameters were affected. For example, total protein of roots (RTP) did not change in Roshan and Shahpasand with Sp7 at optimum water condition, meanwhile, other parameters behave differently (Tables 2 and 4). In contrast, RTP in the tolerant and moderately sensitive pairs of Roshan and Shahpasand with Sp245 were increased due to inoculation. An increase in the protein of plants have been reported in an association of different plant species and bacterial strains ((Vasse et al., 1993; Gamas et al., 1998). For example, different strains of A. brasilense have been caused an addition of total protein in wheat and tomatoes (Saubidet et al., 2002; Mangmang et al., 2015).

PAL and TAL activity in the roots and shoots of Roshan cv. inoculated with Sp245 were increased compare to non-inoculated cultivars at optimum water condition but there was no change in other association pairs except for RPAL in the moderately sensitive pair (Shahpasand-Sp245) which increased by 28\%. A. brasilense Sp245 may enhance the defensive mechanism of wheat cultivars via changing the activities of these enzymes same as other PGPRs, then less reduction in growth parameters was observed in Rhoshan-Sp245 compare to other pairs (Maksimov et al., 2015). There is an evidence that an establishment of a proper relation between plant and bacteria can stimulate the biosynthesis pathway of phenolic compounds as a plant defense mechanism (Vasse et al., 1993). TAL and PAL enzymes are involved in the biosynthesis of phenolic compounds via biosynthesis pathway of phenylpropanoids (Lawton et al., 1983; Nemat Alla et al., 2002). Therefore, increase in the activity of TAL and PAL can cause more phenolic compounds in inoculated plants with a homologous bacterial strain which is an efficient mechanism to deal with the adverse effects of reactive oxygen species during the infection process (Estabrook and Sengupta-Gopalan, 1991). This may be a reason for the less damage to the growth rate in this experiment in inoculated as well as drought conditions.

All growth parameters in non-inoculated tolerant cultivar did not change due to drought condition (except more number of root branches, NRB). However, the most growth parameters in sensitive cultivar were severely decreased. Other reports also show that the reduction in growth parameters in the sensitive cultivars of wheat is more than the tolerant cultivars (Liu et al., 2004). Significant reduction in dry weight and other growth parameters of the sensitive cultivars under drought condition may be due to reduced plant water potential and impairment of mineral absorption and less photosynthesis, protein synthesis and carbohydrate metabolism (Tekle and Alemu, 2016). Among the growth parameters, the number of root branches was increased due to drought in both sensitive and tolerant cultivars with the different rate. Development of an extensive root system in drought condition is an avoidance mechanism to obtain more water from the rhizosphere to protect themselves against drought stress (Liu et al., 
2004). The root and shoot elongation not only was observed in drought condition but also osmotic stress can cause higher root length and also higher activity of PAL and TAL enzymes in wheat seedlings inoculated with A. brasilense strains (Nabti et al., 2010; Hamdia et al., 2004).

Drought condition caused the addition of total protein in root in non-inoculated tolerant cultivar but no change in the sensitive one as compare to optimum water condition. Addition of total protein due to drought stress indicated the role of organic osmolytes in osmotic adjustment for adaptation of plant to drought stress (Serraj and Sinclair, 2002).

Dual effect of inoculation and drought condition simultaneously caused mostly higher growth parameters in Roshan with Sp245 (except for NMR and MSL) as well as with Sp7 (except ARL and NRB), while the growth parameters in Shahpasand cv. (except for NRB) were significantly decreased as compared to non-inoculated cultivars at optimum water condition. Similar studies showed that inoculation of wheat with Sp245 strain increased growth parameters more than Sp7 due to the capability of Sp245 to colonize interior parts of wheat root unlike the Sp7 (Michiels et al., 1991). The Sp245 strain could improve drought tolerance of wheat by the production of ABA, a key phytohormone in plant response to drought stress (Michiels et al., 1991; Cohen et al., 2008).

Dual effect of inoculation and drought condition show that Roshan cv. with Sp245 caused an increase in PAL and TAL activities compared to non-inoculated cultivars at drought or optimum water condition. It has been suggested that inoculated of wheat cultivars with Sp245 has a positive effect on the activity of these enzymes either at drought or optimum water condition. Hence, A. brasilense Sp245 can enhance the defensive mechanism of wheat at the drought condition (Maksimov et al., 2015). PAL and TAL have a main role in drought tolerance in wheat plants through phenylpropanoids biosynthesis pathway and accumulation of more phenolic compounds in plants (Redmann, 1974; Kaur and Zhawar, 2015).

Therefore, it can be concluded that positive effects of inoculation on biochemical properties of the wheat cultivars are mostly related to homology properties of wheat cultivar and bacterial strain not to the sensitivity of cultivar to drought. This is the reason that Roshan (tolerant) and Shahpasand (sensitive) cultivars with Sp245 showed the better results than Sp7. However, the dual effect of inoculation and drought condition represents the interaction effect on the association of wheat cultivar with the strain of bacteria when compared to separate effect.

\section{DREB2 expression of the selected association pairs in different water potentials}

At optimum water condition $\left(\psi_{\mathrm{w}}=0 \mathrm{MPa}\right), D R E B 2$ of inoculated cultivars was down-regulated in the root but up-regulated in the shoot of all selected pairs relative to the respective non-inoculated cultivars. It seems that over-expression of DREB2 in the shoot of inoculated seedlings may be due to the basic effect of A. brasilense strains on the wheat seedling. However, the positive effect in the shoot of tolerant and moderately tolerant pairs is more than sensitive and moderately sensitive pairs. The effect of some other PGPRs such as Arthrobacter protophormiae (SA3), Dietzia natronolimnaea (STR1) and Bacillus subtilis (LDR2) on TaCTR1/TaDREB2 expression in wheat has been shown the enhancement of TaDREB2 gene expression encoding for a transcription factor and improving the tolerance of plants to abiotic stress conditions (Barnawal et al., 2017). 
DREB2 expression in non-inoculated cultivars and the cross-inoculated with A. brasilense strains was also varied under dehydration condition. DREB2 up-regulated in root and shoot of non-inoculated tolerant cv. but did not change in the sensitive cv. due to dehydration. DREB2 up-regulation has been reported in dehydration condition in the tolerant $\mathrm{cv}$. of wheat. For example, Shen and colleagues (2003) reported that drought stress increased TaDREB2 gene expression in tolerant cultivars of wheat. Meanwhile, transgenic RD29A-GmDREB wheat plants had a better tolerance compared to wild type plants which shows the contribution of these two genes in wheat drought tolerance (Shiqing et al., 2005). At 360 minutes after dehydration imposed, DREB2 down-regulated in the root of Roshan and up-regulated in the shoot. As an early gene, DREB2 expression is rapid and transient (peaking at 2 or 3 hours) and then their mRNA levels will decrease (Yamaguchi-Shinozaki and Shinozaki, 2005; Agarwal et al., 2006, 2010). DREB2 down-regulation at longer time of dehydration (360 min) in the tolerant $\mathrm{cv}$. may be due to the transient role of this transcription factor. In contrast, in the sensitive cv., DREB2 up-regulated at this time in the root and down-regulated in the shoot. It seems that in the sensitive cv. at the shorter time $(120 \mathrm{~min})$, dehydration could not make DREB2 up-regulation unless dehydration duration would be longer. Longer dehydration (360 min) means more loss of water potential and more osmotic signals which were produces by the root cells (Taylor et al., 2000; Stein et al., 2000; Rock, 2000; Liu et al., 2005). Likely, the production of signals for stimulation of DREB2 expression was enough only for the roots of sensitive cv. but not for its shoots. This is the reason for DREB2 down-regulation even at $360 \mathrm{~min}$ after dehydration applied.

In this experiment, up-regulation of DREB2 in the tolerant and moderately tolerant pairs and its down-regulation in the sensitive and moderately sensitive pairs at dehydration supported the role of DREB2 gene as an essential early stress-responsive gene in plant tolerance to dehydration (Yamaguchi-Shinozaki and Shinozaki, 2005; Fujita et al., 2011). The early genes encode regulatory proteins such as transcription factors, signal transduction related proteins, osmolytes biosynthesis (Agarwal et al., 2010). There is evidence for better growth of recombinant E. coli cells expressing SbDREB2A (obtained from Salicornia brachiata) in the culture mediums enriched by $\mathrm{NaCl}, \mathrm{PEG}$, and mannitol due to the role of plant's $S b D R E B 2 A$ in the regulation of other stress-regulated genes. This function represents certain interactions between the plant's SbDREB2A with a transcriptional network of the bacterial cells (Gupta et al., 2010). According to our results, it seems that there is probably proper crosstalk between the transcriptional network of A. brasilense Sp245 (the most homologous strain) with Roshan (the most proper cultivar of wheat) which causes highest DREB2 up-regulation in the root of the tolerant pair. DREB2 up-regulation in the root of tolerant pair was more than the shoot which might be due to the localization of A. brasilense Sp245 in the root of Roshan cv. and supports this crosstalk between the transcriptional network of bacteria and plant. DREB2 up-regulation in the root (778-fold) of tolerant pair was more than shoot (1.2-fold) but in the tolerant cv. DREB2 expression was less in the root than the shoot under dehydration condition. This result supports the role of $\mathrm{Sp} 245$ to change $D R E B 2$ expression in the root of Roshan cultivar. In the sensitive cv., unlike the tolerant cv., DREB2 expression did not change.

Dual effects of dehydration and inoculation on DREB2 expression in the tolerant and sensitive pair were different. This effect was positive in the tolerant and moderately tolerant pairs but it was mostly negative or ineffective in the sensitive and moderately sensitive pairs. Meanwhile, the quantity of dual effect was dependent on dehydration 
course. According to our result, it is suggested that inoculation of wheat with A. brasilense $\mathrm{Sp} 245$ can provide a proper tool to improve tolerance of wheat to dehydration via regulation of $D R E B$ expression.

\section{Conclusion}

In conclusion, the effect of inoculation on physiological (growth and biochemical) parameters of wheat seedlings in the sensitive and moderately sensitive pairs was less than the tolerant and moderately tolerant pairs at drought or optimum water condition. The dual effects of dehydration and inoculation on DREB2 expression were positive in the tolerant and moderately tolerant pairs and mostly negative or ineffective in the sensitive and moderately sensitive pairs. However, these effects were depending on the duration of dehydration. It seems that dehydration alone could not cause up-regulation of $D R E B 2$ in non-inoculated wheat cultivars unless the time of stress would be enough meanwhile over-expression of DREB2 in inoculated wheat cultivars with A. brasilense after dehydration may be more due to the effect of the bacteria on wheat seedlings. The authors feel further research is needed to find out the details of the effect of A. brasilense on DREB2 gene expression in wheat including signal transduction pathways and their components using wheat and or A. brasilense mutants.

Acknowledgements. Authors wish to thank Dr. Michael Rothballer from AMP Research Unit MicrobePlant Interactions for providing the bacterial strains. Financial support by the University of Isfahan is gratefully acknowledged.

\section{REFERENCES}

[1] Agarwal, P. K., Agarwal, P., Reddy, M., Sopory, S. K. (2006): Role of DREB transcription factors in abiotic and biotic stress tolerance in plants. - Plant cell reports 25: 1263-1274.

[2] Agarwal, P., Agarwal, P. K., Joshi, A. J., Sopory, S. K., Reddy, M. K. (2010): Overexpression of $P g D R E B 2 A$ transcription factor enhances abiotic stress tolerance and activates downstream stress-responsive genes. - Molecular biology reports 37: 11-25.

[3] Akbari, G. A., Arab, S. M., Alikhani, H., Allakdadi, I., Arzanesh, M. (2007): Isolation and selection of indigenous Azospirillum spp. and the IAA of superior strains effects on wheat roots. - World Journal of Agricultural Sciences 3: 523-529.

[4] Amooaghaie, R., Mostajeran, A., Emtiazi, G. (2004): The effect of compatible and incompatible Azospirillum brasilense strains on proton efflux of intact wheat roots. Plant and Soil 243: 155-160.

[5] Baker, S. S., Wilhelm, K. S., Thomashow, M. F. (1994): The 5'-region of Arabidopsis thaliana cor15a has cis-acting elements that confer cold-, drought-and ABA-regulated gene expression. - Plant molecular biology 24: 701-713.

[6] Baldani, V. L., Baldani, J. I., Döbereiner, J. (1983): Effects of Azospirillum inoculation on root infection and nitrogen incorporation in wheat. - Canadian Journal of Microbiology 29: 924-929.

[7] Baldani, V., Alvarez, M. D. B., Baldani, J., Döbereiner, J. (1986): Establishment of inoculated Azospirillum spp. in the rhizosphere and in roots of field grown wheat and sorghum. - Plant and Soil 90: 35-46. 
[8] Baldani, V., Baldani, J., Döbereiner, J. (1987): Inoculation of field-grown wheat (Triticum aestivum) with Azospirillum spp. in Brazil. - Biology and fertility of soils 4: 37-40.

[9] Barnawal, D., Bharti, N., Pandey, S. S., Pandey, A., Chanotiya, C. S., Kalra, A. (2017): Plant growth-promoting rhizobacteria enhance wheat salt and drought stress tolerance by altering endogenous phytohormone levels and TaCTRI/TaDREB2 expression. Physiologia plantarum 161: 502-514.

[10] Bashan, Y., Holguin, G. (1997): Azospirillum-plant relationships: environmental and physiological advances (1990-1996). - Canadian Journal of Microbiology 43: 103-121.

[11] Beaudoin-Egan, L. D., Thorpe, T. A. (1985): Tyrosine and phenylalanine ammonialyase activities during shoot inhibition in tobacco callus cultures. - Plant Physiology 78: 438441.

[12] Bermner, E., Jenze, H., Gilbertson, C. (1995): Evidence against associative $\mathrm{N}_{2}$ - fixation as a significant source of $\mathrm{N}$ in long-term Wheat plots. - Plant and Soil 175: 13-19.

[13] Bradford, M. M. (1976): A rapid and sensitive method for the quantitation of microgram quantities of protein utilizing the principle of protein dye binding. - Analytical Biochemistry 72: 248-253.

[14] Bray, E. (2000): Response to abiotic stress. - In: Bailey-Serres, J., Weretilnyk, E. (eds.) Biochemistry and molecular biology of plants. USA: Rockville: American Society of Plant Physiologists.

[15] Butt, H. J., Graf, K., Kappl, M. (2006): Physics and chemistry of interfaces. - John Wiley \& Sons.

[16] Casimiro, I., Beeckman, T., Graham, N., Bhalerao, R., Zhang, H., Casero, P., Sandberg, G., Bennett, M. J. (2003): Dissecting Arabidopsis lateral root development. - Trends in plant science 8: 165-171.

[17] Cohen, A. C., Bottini, R., Piccoli, P. N. (2008): Azospirillum brasilense Sp245 produces ABA in chemically-defined culture medium and increases ABA content in Arabidopsis plants. - Plant Growth Regulation 54: 97-103.

[18] Correa-Aragunde, N., Graziano, M., Lamattina, L. (2004): Nitric oxide plays a central role in determining lateral root development in tomato. - Planta 218: 900-905.

[19] Díaz-Zorita, M., Fernández-Canigia, M. V. (2009): Field performance of a liquid formulation of Azospirillum brasilense on dryland wheat productivity. - European journal of soil biology 45: 3-11.

[20] Dobbelaere, S., Vanderleyden, J., Okon, Y. (2003): Plant growth-promoting effects of diazotrophs in the rhizosphere. - Critical reviews in plant sciences 22: 107-149.

[21] Dobereiner, J., Day, J. M. (1976): Association symbiosis in tropical grasses: Characterization of microorganisms and dinitrogen fixing sites. - In: Newton, W. E., Nyman, C. J. (eds.) Proceedings of the first International Sypmosium on Nitrogen Fixation. Pullman: Washington State University Press.

[22] Egawa, C., Kobayashi, F., Ishibashi, M., Nakamura, T., Nakamura, C., Takumi, S. (2006): Differential regulation of transcript accumulation and alternative splicing of a DREB2 homolog under abiotic stress conditions in common wheat. - Genes \& genetic systems 81: 77-91.

[23] El-Khawas, H., Adachi, K. (1999): Identification and quantification of auxins in culture media of Azospirillum and Klebsiella and their effect on rice roots. - Biology and Fertility of Soils 28: 377-381.

[24] El-Samad, A., Hetta, A. (2005): Effect of molybdenum on nitrogenase and nitrate reductase activities of wheat inoculated with Azospirillum brasilense grown under drought stress. - General Applied Plant Physiology 31: 43-54.

[25] Estabrook, E. M., Sengupta-Gopalan, C. (1991): Differential expression of phenylalanine ammonia-lyase and chalcone synthase during soybean nodule development. - The Plant Cell 3: 299-308. 
[26] Fujita, Y., Fujita, M., Shinozaki, K., Yamaguchi-Shinozaki, K. (2011): ABA-mediated transcriptional regulation in response to osmotic stress in plants. - Journal of plant research 124: 509-525.

[27] Gachomo, E. W., Kefela, T., Houngnandan, P., Baba-Moussa, L., Kotchoni, S. O. (2014): Bradyrhizobium japonicum IRAT FA3 increases biomass, yield and drought tolerance in plants. - Journal of Natural Biology 1: 12-23.

[28] Gamas, P., De Billy, F., Truchet, G. (1998): Symbiosis-specific expression of two Medicago truncatula nodulin genes, MtN1 and MtN13, encoding products homologous to plant defense proteins. - Molecular Plant-Microbe Interactions 11: 393-403.

[29] Gupta, K., Agarwal, P. K., Reddy, M., Jha, B. (2010): SbDREB2A, an A-2 type DREB transcription factor from extreme halophyte Salicornia brachiata confers abiotic stress tolerance in Escherichia coli. - Plant Cell Reports 29: 1131-1137.

[30] Hamdia, M. a. E.-S., Shaddad, M., Doaa, M. M. (2004): Mechanisms of salt tolerance and interactive effects of Azospirillum brasilense inoculation on maize cultivars grown under salt stress conditions. - Plant Growth Regulation 44: 165-174.

[31] Hartmann, A., Zimmer, W., Okon, Y. (1994): Physiology of Azospirillum.

[32] Hartmann, A., Baldani, J. I. (2006): The genus Azospirillum. - In: Dworkin, M., Falkow, S., Rosenberg, E., Schleifer, K. H., Stackebrandt, E. (eds.) The prokaryotes Proteobacteria: alpha and beta subclasses New York: Springer.

[33] Hungria, M., Campo, R. J., Souza, E. M., Pedrosa, F. O. (2010): Inoculation with selected strains of Azospirillum brasilense and A. lipoferum improves yields of maize and wheat in Brazil. - Plant and Soil 331: 413-425.

[34] Jain, D. K., Patriquin, D. G. (1984): Root Hair Deformation, Bacterial Attachment, and Plant Growth in Wheat-Azospirillum Associations. - Applied and Environmental Microbiology 48: 1208.

[35] Jakab, G., Ton, J., Flors, V., Zimmerli, L., Métraux, J., Mauch-Mani, B. (2005): Enhancing Arabidopsis salt and drought stress tolerance by chemical priming for its abscisic acid responses. - Plant physiology 139: 267-274.

[36] Jia, H., Zhang, S., Ruan, M., Wang, Y., Wang, C. (2012): Analysis and application of RD29 genes in abiotic stress response. - Acta Physiologia Plantarum 34: 1239-1250.

[37] Kaur, L., Zhawar, V. K. (2015): Phenolic parameters under exogenous ABA, water stress, salt stress in two wheat cultivars varying in drought tolerance. - Indian Journal of Plant Physiology 20: 151-156.

[38] Kramer, P. J., Boyer, J. S. (1995): Water relations of plants and soils. - Academic press.

[39] Krieg, N., Dobereiner, J. (1984): Genus Azospirillum. - In: Nr, K., Jg, H. (eds.) Bergey's Manual of Systematic Bacteriology. London: Williams and Wilkins, Baltimore, MD.

[40] Lawton, M. A., Dixon, R. A., Hahlbrock, K., Lamb, C. J. (1983): Elicitor induction of mRNA activity. Rapid effects of elicitor on phenylalanine ammonia-lyase and chalcone synthase mRNA activities in bean cells. - European Journal of Biochemistry 130(1): 131139.

[41] Liu, H.-S., Li, F. M., Xu, H. (2004): Deficiency of water can enhance root respiration rate of drought-sensitive but not drought-tolerant spring wheat. - Agricultural water management 64: 41-48.

[42] Liu, F., Andersen, M. N., Jacobsen, S. E., Jensen, C. R. (2005): Stomatal control and water use efficiency of soybean (Glycine max L. Merr.) during progressive soil drying. Environmental and Experimental Botany 54: 33-40.

[43] Livak, K. J., Schmittgen, T. D. (2001): Analysis of relative gene expression data using real-time quantitative PCR and the $2^{-\Delta \Delta C T}$ method. - Methods 25: 402-408.

[44] Maksimov, I., Veselova, S., Nuzhnaya, T., Sarvarova, E., Khairullin, R. (2015): Plant growth-promoting bacteria in regulation of plant resistance to stress factors. - Russian Journal of Plant Physiology 62: 715-726.

[45] Mangmang, J. S., Deaker, R., Rogers, G. (2015): Azospirillum brasilense enhances recycling of fish effluent to support growth of tomato seedlings. - Horticulturae 1: 14-26. 
[46] Mcfarland, J. (1907): The nephelometer: an instrument for estimating the number of bacteria in suspensions used for calculating the opsonic index and for vaccines. - Journal of the American Medical Association 49: 1176-1178.

[47] Michiels, K., Croes, C. L., Vanderleyden, J. (1991): Two different modes of attachment of Azospirillum brasilense Sp7 to Wheat roots. - Journal of General Microbiology 137: 2241-2246.

[48] Murashige, T., Skoog, F. (1962): A revised medium for rapid growth and bio assays with tobacco tissue cultures. - Physiologia plantarum 15: 473-497.

[49] Nabti, E., Sahnoune, M., Ghoul, M., Fischer, D., Hofmann, A., Rothballer, M., Schmid, M., Hartmann, A. (2010): Restoration of growth of durum wheat (Triticum durum var. waha) under saline conditions due to inoculation with the rhizosphere bacterium Azospirillum brasilense $\mathrm{NH}$ and extracts of the marine alga Ulva lactuca. - Journal of Plant Growth Regulation 29: 6-22.

[50] Nemat Alla, M. M., Younis, M. E., El-Shihaby, O. A., El-Bastawisy, Z. M. (2002): Kinetin regulation of growth and secondary metabolism in waterlogging and salinity treated Vigna sinensis and Zea mays. - Acta Physiologia Plantarum 24: 19-27.

[51] Okon, Y., Albrecht, S. L., Burris, R. (1977): Methods for growing Spirillum lipoferum and for counting it in pure culture and in association with plants. - Applied and Environmental Microbiology 33: 85-88.

[52] Pakdaman, N., Mostajeran, A., Hojati, Z. (2014): Phosphate concentration alters the effective bacterial quorum in the symbiosis of Medicago truncatula-Sinorhizobium meliloti. - Symbiosis 62: 151-155.

[53] Pereyra, M., Zalazar, C., Barassi, C. (2006): Root phospholipids in Azospirilluminoculated wheat seedlings exposed to water stress. - Plant Physiology and Biochemistry 44: 873-879.

[54] Perrig, D., Boiero, M. L., Masciarelli, O., Penna, C., Ruiz, O. A., Cassan, F. D., Luna, M. V. (2007): Plant-growth-promoting compounds produced by two agronomically important strains of Azospirillum brasilense and implications for inoculant formulation. Applied Microbiology and Biotechnology 75: 1143-1150.

[55] Redmann, R. (1974): Osmotic and specific ion effects on the germination of alfalfa. Canadian Journal of Botany 52: 803-808.

[56] Rock, C. D. (2000): Pathways to abscisic acid-regulated gene expression. - New Phytologist 148: 357-396.

[57] Sakuma, Y., Liu, Q., Dubouzet, J. G., Abe, H., Shinozaki, K., Yamaguchi-Shinozaki, K. (2002): DNA-binding specificity of the ERF/AP2 domain of Arabidopsis DREBs, transcription factors involved in dehydration-and cold-inducible gene expression. Biochemical and biophysical research communications 290: 998-1009.

[58] Santos, R., Hérouart, D., Sigaud, S., Touati, D., Puppo, A. (2001): Oxidative burst in alfalfa-Sinorhizobium meliloti symbiotic interaction. - Molecular plant-microbe interactions 14: 86-89.

[59] Saubidet, M. I., Fatta, N., Barneix, A. J. (2002): The effect of inoculation with Azospirillum brasilense on growth and nitrogen utilization by wheat plants. - Plant and soil 245: 215-222.

[60] Serraj, R., Sinclair, T. (2002): Osmolyte accumulation: can it really help increase crop yield under drought conditions? - Plant, cell and environment 25: 333-341.

[61] Shen, Y. G., Zhang, W. K., He, S. J., Zhang, J. S., Liu, Q., Chen, S. Y. (2003): An EREBP/AP2-type protein in Triticum aestivum was a DRE binding transcription factor induced by cold, dehydration and ABA stress. - Theoritical and Applied Genetics 106: 923-930.

[62] Shiqing, G., Huijun, X., Xianguo, C., Ming, C., Zhaoshi, X., Liancheng, L., Xingguo, Y., Lipu, D., Xiaoyan, H., Youzhi, M. (2005): Improvement of wheat drought and salt tolerance by expression of a stress-inducible transcription factor GmDREB of soybean (Glycine max). - Chinese Science Bulletin 50: 2714-2723. 
[63] Spaepen, S., Vanderleyden, J., Okon, Y. (2009): Plant growth-promoting actions of rhizobacteria. - Advances in botanical research 51: 283-320.

[64] Sriskandarajah, S., Kennedy, I. R., Yu, D., Tchan, Y. T. (1993): Effects of plant growth regulators on acetylene-reducing associations between Azospirillum brasilense and wheat. - Plant and soil 153: 165-178.

[65] Stein, N., Feuillet, C., Wicker, T., Schlagenhauf, E., Keller, B. (2000): Subgenome chromosome walking in wheat: a $450-\mathrm{kb}$ physical contig in Triticum monococcum L. spans the Lr10 resistance locus in hexaploid wheat (Triticum aestivum L.). - Proceedings of the National Academy of Sciences 97: 13436-13441.

[66] Stets, M., Alqueres, S., Souza, E., Schmid, M., Hartmann, A., Cruz, L. (2015): Quantification of Azospirillum brasilense FP2 Bacteria in Wheat Roots by Strain-Specific Quantitative PCR. - Applied and environmental microbiology 81: 6700-6709.

[67] Sumner, M. (1990): Crop responses to Azospirillum inoculation. - Advances in Soil Science 12: 53-123.

[68] Sweet, H. C., Bolton, W. E. (1979): The surface decontamination of seeds to produce axenic seedlings. - American Journal of Botany 66: 692-698.

[69] Taylor, I. B., Burbidge, A., Thompson, A. J. (2000): Control of abscisic acid synthesis. Journal of Experimental Botany 51: 1563-1574.

[70] Tekle, A., Alemu, M. (2016): Drought Tolerance Mechanisms in Field Crops. - World Journal of Biology and Medical Sciences 3: 19-39.

[71] Thomson, W. (1872): On the equilibrium of vapour at a curved surface of liquid. Proceedings of the Royal Society of Edinburgh 7: 63-68.

[72] Vasse, J., De Billy, F., Truchet, G. (1993): Abortion of infection during the Rhizobium meliloti-alfalfa symbiotic interaction is accompanied by a hypersensitive reaction. - The Plant Journal 4: 555-566.

[73] Veresoglou, S. D., Menexes, G. (2010): Impact of inoculation with Azospirillum spp. on growth properties and seed yield of wheat: a meta-analysis of studies in the ISI Web of Science from 1981 to 2008. - Plant and Soil 337: 469-480.

[74] Yamaguchi-Shinozaki, K., Shinozaki, K. (2005): Improving drought and cold-stress tolerance in transgenic rice. Rice is life: scientific perspectives for the 21st century. Proceedings of the World Rice Research Conference held in Tsukuba, Japan, 4-7 November 2004, 2005. International Rice Research Institute (IRRI), 94-97.

[75] Yang, J., Kloepper, J. W., Ryu, C.-M. (2009): Rhizosphere bacteria help plants tolerate abiotic stress. - Trends in plant science 14: 1-4. 


\section{APPENDIX}

Appendix 1. The averages of ranking numbers in the associated pairs between wheat (Triticum aestivum L.) cultivars (Omid, Roshan, Sardari, Shahpasand, Shoaleh and Tabasi) and two strains of A. brasilense (Sp7, Sp245) at optimum water content (80\% FC) and drought condition (40\% FC) based on growth and biochemical parameters. At 80\% FC, the minimum and maximum numbers show the most compatible (homologous) and most noncompatible (heterologous) association pairs, respectively; At 40\% FC, the minimum and maximum numbers show the most tolerant and most sensitive association pairs to drought, respectively; At 40\% FC, Tabasi cultivar is not included due to lack of its successful association with both bacterial strains under optimum water condition) (NI: non-inoculated)

\begin{tabular}{|c|c|c|c|}
\hline \multicolumn{2}{|c|}{ Pairs } & \multicolumn{2}{|c|}{$\begin{array}{l}\text { Ranking number } \\
\end{array}$} \\
\hline Wheat cultivars & A.brasilense strains & $80 \%$ FC & $40 \% \mathrm{FC}$ \\
\hline \multirow[t]{3}{*}{ Omid } & Non-inoculated & 8.2 & 8.5 \\
\hline & Sp7 & 6.3 & 7.2 \\
\hline & $\mathrm{Sp} 245$ & 4.5 & 5 \\
\hline \multirow[t]{3}{*}{ Sardari } & Non-inoculated & 12.2 & 10 \\
\hline & Sp7 & 9 & 7.3 \\
\hline & $\mathrm{Sp} 245$ & 7.7 & 5.5 \\
\hline \multirow[t]{3}{*}{ Roshan } & Non-inoculated & 6.5 & 4 \\
\hline & Sp7 & 3.3 & 2.7 \\
\hline & $\mathrm{Sp} 245$ & 3 (Minimum) & 1 (Minimum) \\
\hline \multirow[t]{3}{*}{ Sloaleh } & Non-inoculated & 13.8 & 15.0 \\
\hline & Sp7 & 10.3 & 14 \\
\hline & $\mathrm{Sp} 245$ & 7.5 & 11.2 \\
\hline \multirow[t]{3}{*}{ Tabasi } & Non-inoculated & 12.5 & - \\
\hline & Sp7 & 10.5 & - \\
\hline & $\mathrm{Sp} 245$ & 10.5 & - \\
\hline \multirow[t]{3}{*}{ Shahpasand } & Non-inoculated & 12.2 & 10.8 \\
\hline & Sp7 & 11 (Maximum) & 16 (Maximum) \\
\hline & $\mathrm{Sp} 245$ & 8 & 11.7 \\
\hline
\end{tabular}

